\title{
Regional anesthesia, block room and efficiency: putting things in perspective
}

Pierre Drolet MD FRCPC, Michel Girard MD MHPE FRCPC

I $\mathrm{N}$ this issue of the Journal, Armstrong and Cherry report on the impact of using upper limb regional anesthesia on the different time intervals measured during the operative process. ${ }^{l}$ Of particular interest is their conclusion that a block room (BR) devoted to the preoperative realization of regional techniques reduces the presurgical time in the actual operating room (OR). Although it seems self-explanatory that time spent performing the blockade in the BR will somehow allow for a shorter interval in the OR before the start of the surgery, the magnitude of the time saved and its cost need to be considered before these study results are used to advocate the creation of bona fide BRs in most hospitals. Because of the numerous variables and individuals ${ }^{2}$ implicated in the operative process, only accurate data collection followed by interdisciplinary teamwork can be expected to enhance its efficiency significantly. ${ }^{3}$ Still, it is interesting to examine the details of the process in order to pinpoint which elements can be modified and result in a positive impact. Is the presurgical time spent on anesthesia a legitimate target for OR managers striving to increase the performance of their suite? How likely are we to improve the efficiency of the operative process by asking anesthesiologists to try to shorten that period? Dexter et al., using a computer simulation, studied the decrease in case duration required to complete an additional case during regular hours in an OR suite. ${ }^{4}$ The decreases in each case's duration required to create enough new open OR time to reliably schedule another case were 30-39, 79-110 and 105-110 min for cases of a mean duration of one, two and three hours respectively. The model applied to an OR suite with one to 15 ORs. Since the magnitude of the time savings that can be expected following different modifications of the anesthesia technique, whether regional or general, are in the 13 to 23 min range ${ }^{1,5,6}$ it may be unrealistic to expect that demands involving the anesthesiology staff alone will bring significant improvement in OR performance. In fact, a report using an actual case series of 709 consecutive patients undergoing a mix of 11 different elective operations at a tertiary care centre showed that anesthesia-controlled time would have to be decreased by more than $100 \%$ to permit one additional 30-min surgery to be performed after an eighthour workday if the previous operations all lasted at least 45 min. $^{7}$ Naturally, OR suites where longer and more complex procedures are performed on a regular basis are the least likely to benefit from reductions in turnover times between cases. ${ }^{8}$ Nonetheless, some authors have succeeded at increasing the number of cases performed following the implementation of measures targeting the anesthesia turnover time. ${ }^{5}$ Sokolovic et al. found that increasing anesthesia staffing by one attending physician and one nurse allowed for the addition of about 0.6 case. day $^{-1}$ in an OR suite in which a mean of 162 operations. month $^{-1}$ were performed, and where the most frequent surgery was termination of pregnancy, a fairly short procedure. Is it really surprising to find that increasing human and therefore financial resources may lead to some increase in the number of patients treated? In a report on the effects of regional and general techniques on anesthesia-controlled time in outpatient knee surgery, Williams et al. studied an institutional database including 369 patients undergoing anterior cruciate ligament reconstruction. ${ }^{9}$ They concluded that, compared to general or combined general-regional anesthesia, proceeding with a regional technique in a $\mathrm{BR}$ decreased the anesthesia time in the OR by less than nine minutes.case ${ }^{-1}$ on average. As stated earlier,

From the Department of Anesthesiology, Maisonneuve-Rosemont Hospital, Montreal, Quebec, Canada.

Address correspondence to: Dr. Pierre Drolet, Department of Anesthesiology, Maisonneuve-Rosemont Hospital, 5415, l'Assomption blvd, Montreal, Quebec HIT 2M4, Canada. Phone: 514-252-3426; Fax: 514-252-3542; E-mail: pdrolet@aei.ca 
it is unlikely that such savings will be sufficient to schedule even one additional short case during a regular day. In that study, the lone anesthesiologist in charge had the luxury of proceeding with regional anesthesia on the patient waiting for surgery, assisted by a nurse anesthetist, while another nurse anesthetist cared for the patient actually undergoing surgery. In their conclusion, the authors stated that, although associated with the lowest anesthesia-controlled time, regional anesthesia with a BR produced additional costs that had to be weighed in the equation.

Looking closely at the results of Armstrong and Cherry $^{1}$ in this issue, we notice that amongst the 1,023 upper limb cases identified as valid for analysis during the six-month period, $319(31.2 \%)$ received regional anesthesia, either alone or combined with general anesthesia, and only 151 of those cases benefited from the BR. Why was the BR not used more systematically? We suspect that reasons are numerous and all too familiar to most clinicians practicing in a busy environment. Unavailability of qualified personnel to care for the patient still in the OR, the next patient not being ready (for whatever reason) at the moment that would be most convenient for us or last minute changes in the schedule are only a few of the reasons that might explain why less than half of the blockades in Armstrong and Cherry's report were performed in the BR. Moreover, the first cases of the morning do not need to go through the BR since the OR is unoccupied. It is also difficult to advocate the use of the BR outside regular hours since most hospitals do not provide the necessary resources around the clock. So, even in a specialized environment such as the authors' hospital, the BR ends up being used for an estimated 300 cases of upper limb surgery each year. Of course, other types of regional anesthesia may be performed in the BR but, if we consider only upper limb surgery, the facilities would be used, on average, barely more than once a day at the most. Even if all upper limb blockades in the authors' hospital were to be performed in the BR, the room would be used only twice a day. Of course, such figures make little sense from a cost/effectiveness point of view, unless the facilities doubled as a surveillance unit of some sort. The authors' centre also use it for ophtalmology patients; other hospitals will elect to combine the BR with the recovery room. Another solution is to set up the BR on a temporary basis. If most of the cases involving the performance of regional techniques can be grouped and scheduled on a specific day, dedicating resources exclusively to the BR for that particular day might then be worthwhile, provided the cases are of short duration. So, unless a hospital caters for a very specialized population in need mostly of short duration surgery under regional anesthesia, setting up a permanently staffed BR dedicated exclusively to the preoperative realization of singleinjection or continuous blocks would seem unrealistic in this era of cost-containment. Does it mean that the advantages of proceeding with the realization of a regional technique in a calm environment without the pressure added by an anxious surgical team manifesting its readiness to start should be disregarded? Of course not, the positive elements identified by Armstrong and Cherry with regard to the «soak time» of blocks and the easiness provided by the BR for the teaching of regional anesthesia need to be emphasized. In fact, finding and setting up a place that can be used, when needed, as a BR with little additional costs, can facilitate our work as long as the logistic problems associated to the preoperative aspect of the block (availability of staff and patients) do not overwhelm us.

Armstrong and Cherry also argue that regional anesthesia can help streamlining the postoperative process. Although such an argument has been proposed for lower limb surgery, ${ }^{10}$ it can be applied more convincingly to upper limb operations since most clinicians deem adequate to send home patients with an arm still partially blocked. ${ }^{11}$ Then, is regional anesthesia really plagued with the dual role of being a positive asset in the postoperative period while being a source of delays and problems during the preoperative process or are those images only false perceptions? After performing a meta-analysis of 26 randomized trials (including a total of 1,874 patients), Dexter concluded that, on average, the type of anesthesia (regional vs general) had no significant impact on the surgical time, but the methods used by the author excluded the anesthesia-controlled time. ${ }^{12}$ The time needed for various regional techniques may differ significantly, some techniques (spinal, in regional anesthesia) being considered by many as fast as general anesthesia. Plexus and peripheral nerve blocks and, to a lesser extent, epidural blocks, are usually perceived as the most time-consuming techniques. ${ }^{6}$ Considering their benefits for patients, ${ }^{13,14}$ is the BR the solution to make those techniques more acceptable to all (anesthesiologists, surgeons and OR managers) in most hospitals? Yes... and no. The answer is yes if our goal is to provide an environment where the techniques can be performed and taught in an optimal and serene manner with less pressure. Still, we have to be able to set it up with minimal costs and work in an environment with enough trained staff to assist us do both: care for one patient while proceeding with the block on the next one. Unfortunately, the answer is no if what we expect is a significant increase in performance and a reliable way to do more cases. 


\section{Anesthésie régionale, salle de bloc et efficacité : perspectives}

Dans le présent numéro du Journal, Armstrong et Cherry rendent compte de l'effet de l'utilisation de l'anesthésie régionale du membre supérieur sur les différents intervalles de temps mesurés pendant le processus opératoire. ${ }^{1}$ Dans leur conclusion, les auteurs affirment que si on réserve une salle de bloc $(\mathrm{SB})$ à la réalisation préopératoire de techniques régionales, on peut réduire le temps préchirurgical en salle d'opération (SO) proprement dit. Bien qu'il semble évident que le temps passé à administrer le bloc dans la SB permette d'une manière ou d'une autre de raccourcir l'intervalle qui précède le début de l'opération en SO, l'importance du temps épargné et le coût de cette entreprise doivent être considérés avant de penser à utiliser les résultats de cette étude pour légitimer la création de SBs dans la majorité des hôpitaux. Étant donné le nombre de variables et de personnes ${ }^{2}$ impliquées dans une intervention chirurgicale, seule une cueillette de données précise supervisée par une équipe de travail interdisciplinaire qui en assure le suivi peut accroître significativement son efficacité. ${ }^{3}$ Toutefois, il est intéressant de vérifier les détails du processus dans le but d'indiquer quels éléments peuvent être modifiés et produire un impact positif. Est-ce que le temps préchirurgical passé à administrer l'anesthésie est une cible légitime pour les responsables de la SO qui s'efforcent d'augmenter la performance de leur unité ? Allons-nous vraiment améliorer l'efficacité du processus opératoire en demandant aux anesthésiologistes d'essayer de réduire cette période ? Dexter et coll. ont étudié, à l'aide d'une simulation informatisée, la réduction du temps par cas nécessaire pour permettre d'ajouter un cas supplémentaire pendant les heures régulières de la $\mathrm{SO} .{ }^{4} \mathrm{La}$ réduction du temps de chaque cas, nécessaire pour créer suffisamment d'espace en SO pour accepter un autre cas de façon certaine, a été de 30-39, 79-110 et 105-110 min pour des cas d'une durée moyenne de une, deux et trois heures, respectivement. Le modèle a été appliqué à un bloc opératoire de 15 SOs. Comme l'importance attendue du temps épargné après avoir fait différentes modifications à la technique anesthésique, qu'elle soit régionale ou générale, se situe entre 13 et $23 \mathrm{~min},{ }^{1,5,6}$ il serait irréaliste de penser que des modifications impliquant seulement le personnel d'anesthésie apportent des améliorations importantes de la performance de la SO. De fait, un rapport utilisant une série de 709 patients consécutifs devant subir un ensemble de 11 différentes opérations réglées, dans un centre de soins tertiaires, a montré que le temps exclusivement anesthésique devrait être réduit de plus de $100 \%$ pour permettre de réaliser une opération supplémentaire de 30 min après une journée de travail de huit heures si les opérations précédentes duraient toutes au moins 45 min. ${ }^{7}$ Naturellement, les salles d'opération où des interventions plus longues et plus complexes sont réalisées sur une base régulière sont les moins susceptibles de bénéficier de ces réductions du temps de rotation entre les cas. ${ }^{8}$ Néanmoins, certains auteurs ont réussi à augmenter le nombre de cas opérés à la suite de l'application de mesures visant le temps de rotation de l'anesthésie. ${ }^{5}$ Sokolovic et coll. ont trouvé qu'en augmentant le personnel d'anesthésie d'un médecin traitant et d'une infirmière, on pouvait ajouter environ 0,6 cas.jour ${ }^{-1}$ dans un bloc opératoire où une moyenne de 162 opérations. mois $^{-1}$ étaient réalisées, et où l'opération la plus fréquente était l'interruption de grossesse, une opération plutôt rapide. Est-ce réellement surprenant de découvrir que l'ajout de ressources humaines et, par conséquent, financières, puissent conduire à une augmentation du nombre de patients traités ? Dans un article sur les effets des techniques régionale et générale sur le temps exclusivement anesthésique en chirurgie ambulatoire du genou, Williams et coll. ont étudié la banque de données d'un établissement incluant 369 patients devant subir une reconstruction du ligament croisé antérieur. ${ }^{9}$ Ils concluent que, comparée à l'anesthésie générale ou à l'anesthésie générale-régionale combinée, la technique régionale utilisée en $\mathrm{SB}$ diminue le temps d'anesthésie en SO par moins de neuf minutes. cas $^{-1}$ en moyenne. Comme nous l'avons déjà indiqué, il est peu probable que cette économie soit suffisante pour permettre d'ajouter un cas de plus à l'horaire régulier d'une journée. Dans l'étude, l'unique anesthésiologiste traitant avait la chance de procéder à l'anesthésie régionale du patient en attente de son opération, assisté par une infirmière, pendant qu'une autre infirmière s'occupait du patient déjà en SO. Dans leur conclusion, les auteurs déclarent que, même si elle est associée à un temps anesthésique plus court, l'anesthésie régionale avec SB engendre un coût supplémentaire qui doit être considéré dans l'équation.

En examinant les résultats d'Armstrong et Cherry ${ }^{1}$ de plus près, nous notons que parmi les 1023 cas d'opération des membres supérieurs validés pour l'analyse pendant la période de six mois, 319 (31,2 \%) patients ont reçu une anesthésie régionale, soit seule, 
soit combinée à une anesthésie générale, et $15 \mathrm{l}$ seulement ont bénéficié de la SB. Pourquoi la SB n'a-t-elle pas été utilisée plus systématiquement ? Nous croyons que les raisons sont nombreuses et que trop familières pour les cliniciens qui exercent dans un milieu aux horaires chargés. La non-disponibilité de personnel qualifié pour s'occuper du patient encore en $\mathrm{SO}$, le patient suivant qui n'est pas prêt (pour une raison ou une autre) au moment qui nous conviendrait davantage ou des changements de dernière minute à l'horaire ne sont que quelques-unes des raisons qui peuvent expliquer pourquoi moins de la moitié des blocs de l'étude d'Armstrong et Cherry ont été réalisés dans la SB. Qui plus est, les premiers cas de la journée n'ont pas besoin de passer par la SB, puisque la SO n'est pas occupée. C'est aussi difficile de conseiller l'usage de la SB en dehors des heures régulières, car la plupart des hôpitaux ne fournissent pas les ressources nécessaires pendant 24 h. Donc, même dans un milieu spécialisé comme l'hôpital où travaillent les auteurs, la SB n'est finalement utilisée que pour 300 cas prévus d'opération des membres supérieurs chaque année. Bien sûr, des techniques d'anesthésie autres que régionales peuvent être utilisées en $\mathrm{SB}$, mais si nous ne tenons compte que des opérations des membres supérieurs, la salle serait occupée, en moyenne, à peine plus d'une fois par jour tout au plus. À l'hôpital des auteurs, même si tous les blocs des membres supérieurs étaient faits dans la SB, la salle ne serait utilisée que deux fois par jour. Naturellement, de tels chiffres n'ont pas beaucoup de sens du point de vue de la rentabilité, à moins que les installations ne servent aussi d'unité de surveillance pour une autre clientèle. Au centre des auteurs, on l'utilise aussi pour les patients d'ophtalmologie ; dans d'autres hôpitaux, on choisit de combiner la SB et la salle de réveil. Une autre solution est d'installer une SB temporaire. Si la majorité des cas appelant à une technique régionale peuvent être regroupés et prévus pour une journée en particulier, il pourrait être très intéressant d'accorder des ressources exclusivement pour la SB ce jour-là, pourvu que les cas soient de courte durée. Ainsi, à moins que la clientèle d'un hôpital n'ait des besoins très spécialisés pour des interventions de courte durée sous anesthésie régionale, l'installation permanente d'une $\mathrm{SB}$, avec son personnel, dédiée exclusivement à la réalisation préopératoire de blocs simples ou continus semble irréaliste à une époque de réduction des dépenses. Est-ce à dire que l'avantage d'administrer une anesthésie régionale dans un environnement calme, sans la pression ajoutée de l'équipe chirurgicale impatiente, prête à commencer l'opération, doit être rejeté ? Certainement pas. Les éléments positifs identifiés par Armstrong et Cherry, relatifs à la "durée d'imprégnation» des blocs et à la facilité procurée par la SB d'enseigner l'anesthésie régionale, doivent être soulignés. De fait, trouver et aménager un endroit qu'on peut utiliser, au besoin, comme une SB avec des coûts supplémentaires minimes, peut faciliter le travail en autant que les problèmes logistiques associés à l'aspect préopératoire du bloc (disponibilité du personnel et des patients) ne sont pas insurmontables.

Armstrong et Cherry déclarent également que l'anesthésie régionale peut aider à simplifier le processus postopératoire. Cet argument a été proposé pour les opérations des membres inférieurs, ${ }^{10}$ mais il peut encore mieux s'appliquer aux opérations des membres supérieurs, car la plupart des cliniciens jugent approprié de laisser sortir les patients même s'ils ont un bras encore partiellement anesthésié. ${ }^{11}$ Alors, l'anesthésie régionale est-elle réellement condamnée à jouer un double rôle, celui d'un atout positif en période postopératoire et celui de responsable des retards et des problèmes pendant le processus préopératoire, ou ces images ne sont-elles que de fausses perceptions? Après une méta-analyse de 26 essais randomisés (comprenant l 874 patients), Dexter a conclu qu'en moyenne, le genre d'anesthésie (régionale ou générale) n'avait pas d'impact significatif sur le temps chirurgical, mais les méthodes utilisées par l'auteur ne tenaient pas compte $\mathrm{du}$ temps exclusivement anesthésique. ${ }^{12}$ Le temps nécessaire à l'exécution de diverses techniques régionales peut varier de façon significative ; beaucoup considèrent certaines techniques, comme l'anesthésie rachidienne et régionale $i v$, aussi rapides que l'anesthésie générale. Les blocs de plexus et de nerfs périphériques et, dans une moindre mesure, les blocs périduraux, sont habituellement perçus comme les techniques les plus longues. ${ }^{6} \mathrm{Si}$ on pense aux avantages de ces techniques pour les patients, ${ }^{13,14}$ est-ce que la SB serait le moyen de les rendre plus acceptables pour tous, anesthésiologistes, chirurgiens et responsables de la SO, dans une majorité d'hôpitaux ? Oui... et non. La réponse est oui si notre objectif est de fournir un environnement où les techniques peuvent être réalisées et enseignées d'une manière optimale et détendue avec le moins de pression possible. Toutefois, il faut pouvoir aménager la SB au coût le plus bas et travailler dans un milieu où le personnel qualifié, et en nombre suffisant, nous assiste pour faire les deux : continuer la prise en charge d'un premier patient tout en procédant au bloc chez un autre patient. Malheureusement, la réponse est non si ce que nous attendons de la mise en fonction d'une SB est une augmentation significative de la performance et un moyen fiable d'opérer plus de patients. 


\section{References}

1 Armstrong KPJ, Cherry RA. Brachial plexus anesthesia compared to general anesthesia when a block room is available. Can J Anesth 2004; 51: 41-5.

2 Strum DP, Sampson AR, May JH, Vargas LG. Surgeon and type of anesthesia predict variability in surgical procedure times. Anesthesiology 2000; 92: 1454-66.

3 Overdyk FJ, Harvey SC, Fishman RL, Shippey F. Successful strategies for improving operating room efficiency at academic institutions. Anesth Analg 1998; 86: 896-906.

4 Dexter F, Macario A. Decrease in case duration required to complete an additional case during regularly scheduled hours in an operating room suite: a computer simulation study. Anesth Analg 1999; 88: 72-6.

5 Sokolovic E, Hiro P, Wyss $P$, et al. Impact of the reduction of anaesthesia turnover time on operating room efficiency. Eur J Anaesthesiol 2002; 19: 560-3.

6 Chan VW, Peng PW, Kaszas Z, et al. A comparative study of general anesthesia, intravenous regional anesthesia, and axillary block for outpatient hand surgery: clinical outcome and cost analysis. Anesth Analg 2001; 93: 1181-4.

7 Dexter F, Coffin S, Tinker JH. Decreases in anesthesiacontrolled time cannot permit one additional surgical operation to be reliably scheduled during the workday. Anesth Analg 1995; 81: 1263-8.

8 Mazzei WJ. Operating room start times and turnover times in a university hospital. J Clin Anesth 1994; 6: 405-8.

9 Williams BA, Kentor ML, Williams JP, et al. Process analysis in outpatient knee surgery. Effects of regional and general anesthesia on anesthesia-controlled time. Anesthesiology 2000; 93: 529-38.

10 Williams BA, DeRiso BM, Engel LB, et al. Benchmarking the perioperative process: II. Introducing anesthesia clinical pathways to improve processes and outcomes and to reduce nursing labor intensity in ambulatory orthopedic surgery. J Clin Anesth 1998; 10: 561-9.

11 Klein SM, Nielsen KC, Greengrass RA, et al. Ambulatory discharge after long-acting peripheral nerve blockade: 2382 blocks with ropivacaine. Anesth Analg 2002; 94: 65-70.

12 Dexter $F$. Regional anesthesia does not significantly change surgical time versus general anesthesia-a metaanalysis of randomized studies. Reg Anesth Pain Med 1998; 23: 439-43.

13 Ilfeld BM, Morey TE, Enneking FK. Continuous infraclavicular brachial plexus block for postoperative pain control at home. A randomized, double-blinded, placebo-controlled study. Anesthesiology 2002; 96 : 1297-304
14 Krone SC, Chan VW, Regan J, et al. Analgesic effects of low-dose ropivacaine for interscalene brachial plexus block for outpatient shoulder surgery-a dose-finding study. Reg Anesth Pain Med 2001; 26: 439-43. 